\title{
Le Syndrome Du Canal Carpien À Lomé (Togo)
}

\author{
Fianyo E., \\ Service de Rhumatologie, CHR Lomé Commune, \\ Université de Lomé-Togo \\ Kakpovi K., \\ Service de Rhumatologie, CHR Tomdè-Kara, \\ Université de Kara-Togo \\ Gbeassor-Komlanvi F., \\ Département de santé Publique, \\ Université de Lomé, Togo \\ Tagbor $K$., \\ Service de Rhumatologie, Hôpital de Bè, \\ Université de Lomé-Togo \\ Houzou P., \\ Service de Rhumatologie, \\ CHU Kara, Université de Kara-Togo \\ Koffi-Tessio V.E.S., \\ Oniankitan $O$., \\ Mijiyawa M., \\ Service de Rhumatologie, CHU Sylvanus Olympio, \\ Université de Lomé-Togo
}

Doi:10.19044/esj.2020.v16n9p229 URL:http://dx.doi.org/10.19044/esj.2020.v16n9p229

\section{Résumé}

Objectif: Décrire les caractéristiques sociodémographiques, cliniques et thérapeutiques des patients souffrant d'un syndrome du canal carpien à Lomé, au Togo. Patients et Méthode: Il s'est agi d'une étude transversale menée sur dossiers du 1er janvier 2011 au 31 décembre 2018 dans les trois Services Hospitaliers de Rhumatologie de Lomé (Togo). Résultats: Cent quatre-vingt-douze patients (166 femmes, 126 hommes) ont présenté un syndrome du canal carpien pendant la période d'étude. Leur âge moyen était de 54, $2 \pm 13$ ans (extrêmes: 26 et 83 ans). L'indice de masse corporelle moyen était de $28,8 \pm 6,1 \mathrm{~kg} / \mathrm{m}^{2}$ (extrêmes: 18 et 55,4 ). Le sexe féminin, l'obésité $(\mathrm{p}=0,001)$, et le travail prolongé à l'ordinateur $(\mathrm{p}=0,005)$ ont été identifiés comme des facteurs de risque de SCC. Les manifestations cliniques ont été dominées par les paresthésies à type de fourmillements $(77,1 \%$ des 
patients) et la douleur (57,8\% des patients). L'atteinte était bilatérale chez 120 patients $(62,5 \%)$. Cent neuf patients $(56,8 \%)$ ont présenté un signe de Tinel, et 39 patients $(20,3 \%)$ ont présenté un signe de Phalen associé au signe de Tinel. La presence du signe de Tinel était corrélée à l'obésité $(p=0,001)$. Le traitement était représenté par les infiltrations locales de cortioïdes (147 patients, 76,6\%), les antalgiques et les antiinflammatoires non stéroïdiens (104 patients, 54,2\%). Conclusion: Le SCC à Lomé est habituellement idiopathique. Le sexe féminin, l'obésité, et le travail prolongé à l'ordinateur sont des facteurs de risque. Les infiltrations locales de corticoïdes sont efficaces.

Mots clefs: Syndrome Du Canal Carpien, Diagnostic, Traitement, Afrique Noire 


\title{
Carpal Tunnel Syndrome Among Rheumatologic Patients In Lomé (Togo)
}

\author{
Fianyo E., \\ Service de Rhumatologie, CHR Lomé Commune, \\ Université de Lomé-Togo \\ Kakpovi K., \\ Service de Rhumatologie, CHR Tomdè-Kara, \\ Université de Kara-Togo \\ Gbeassor-Komlanvi F., \\ Département de santé Publique, \\ Université de Lomé, Togo \\ Tagbor $K$., \\ Service de Rhumatologie, Hôpital de Bè, \\ Université de Lomé-Togo

\section{Houzou P.,} \\ Service de Rhumatologie, \\ CHU Kara, Université de Kara-Togo \\ Koffi-Tessio V.E.S., \\ Oniankitan $\mathrm{O}$., \\ Mijiyawa M., \\ Service de Rhumatologie, CHU Sylvanus Olympio, \\ Université de Lomé-Togo
}

\begin{abstract}
Objective: To describe the socio-demographic, clinical and therapeutic characteristics of patients with carpal tunnel syndrome (CTS) in Lomé (Togo). Patients and methods: This was a cross-sectional study. Data were collected from $1^{\text {st }}$ of January 2011 to $31^{\text {st }}$ of December 2018 in three Rheumatologic Hospital Wards in Lomé. Results: One hundred and ninetytwo patients were diagnosed with CTS (166 women and 26 men). Their average age was between $54.2 \pm 13$ years (range from 26 to 83 years). The average body mass index of male and female patients was $28,8 \pm 6,1 \mathrm{~kg} / \mathrm{m}^{2}$ (range 18 and 55.4). Female gender, obesity $(\mathrm{p}=0,001)$, and prolonged computer use $(\mathrm{p}=0,005)$ were identified as key risk factors. The main symptoms were: tingling paresthesia $(77.1 \%)$ and pain $(57.8 \%)$. The symptoms were bilateral in 120 patients $(62.5 \%)$. The Tinel sign was positive
\end{abstract}


in 109 patients $(56.8 \%)$, and 39 patients $(20,3 \%)$ had both the Tinel sign and the Phalen sign. The Tinel sign was related to obesity $(\mathrm{p}=0,001)$. Management of CTS was performed by local steroid injections (147 patients, 76,6\%), pain killers and NSAID (104 patients, 54,2\%). Conclusion: CTS is usually idiopathic in Lomé. Female gender, obesity and prolonged computer use are risk factors. Local infiltration of steroids is an effective treatment.

Keywords: Carpal Tunnel Syndrome, Diagnosis, Treatment, Black Africa

\section{Introduction}

Le syndrome du canal carpien est l'ensemble des signes traduisant la souffrance du nerf médian dans le canal carpien, du fait d'une compression ou d'un étirement. C'est le plus fréquent des syndromes canalaires. Le diagnostic repose sur l'existence de douleurs et/ou de paresthésies des trois premiers doigts, d'un signe de Tinel et/ou du signe de Phalen. La douleur peut irradier vers l'avant-bras. L'électromyogramme est nécessaire avant une intervention chirurgicale. Il permet d'éliminer d'autres diagnostics, et d'apprécier la sévérité de la neuropathie canalaire (Mazières, 2018; Allaya, 2019; LebrunGrandié, 2019). Dans les formes évoluées un déficit sensitif et moteur peut s'installer avec apparition de maladresses. En fonction de la sévérité de l'atteinte les conséquences du syndrome du canal carpien peuvent aller du simple inconfort, à la perte d'emploi, ou à une altération significative de la qualité de vie (Shin, 2018; Aouatef, 2016). Ce syndrome est habituellement idiopathique. Il affecte préférentiellement les femmes (Mazières, 2018). Lee a trouvé une prévalence qui augmentait avec l'âge. Les "cols bleus" étaient plus exposés que les "cols blancs" (Lee, 2019).

Le traitement des formes non déficitaires repose sur les infiltrations de corticoïdes et les attelles. Une deuxième infiltration peut être réalisée un mois après la première, si celle-ci n'a pas été efficace. Il est déconseillé de réaliser plus de trois infiltrations (Chammas, 2014). Luchetti recommande d'associer la rééducation fonctionnelle et des agents neurotrophiques tel que l'acide alpha-lipoïque ((Luchetti, 2017).

L'objectif de ce travail a été de décrire les caractéristiques sociodémographiques, cliniques et thérapeutiques des patients souffrant d'un syndrome du canal carpien à Lomé, au Togo.

\section{Patients et méthode}

Il s'est agi d'une étude transversale sur dossiers de consultation réalisée du $1^{\mathrm{er}}$ janvier 2011 au 31 décembre 2018 dans les trois services de rhumatologie de Lomé. Le Service de Rhumatologie du Centre HospitaloUniversitaire Sylvanus Olympio accueille en moyenne 1000 patients en 
consultation chaque année, celui de l'Hôpital de Bè et le Centre Hospitalier Régional Lomé Commune en accueillent chacun 600.

Ont été inclus tous les patients pour lesquels a été posé le diagnostic de syndrome du canal carpien. L'âge, le sexe, la profession, les antécédents pathologiques et comorbidités, les signes cliniques, les traitements administrés et leurs résultats ont été recueillis pour chaque patient. Le diagnostic a été clinique. L'électromyogramme n'a été réalisé chez aucun patient. L'existence de facteurs de risque a reposé sur l'interrogatoire. Ont été considérés comme travailleurs manuels (caissière, couturière, tresseuse/coiffeuse, cuisinière, électricien, boulangère, domestique, photographe) tous ceux réalisant des mouvements répétitifs de flexion/extension ou torsion du poignet ou des doigts; et comme travailleurs de force (agriculteur, maçon, mécanicien, boucher, menuisier, carreleur, ouvrier, plombier, militaire) ceux mettant en jeu la force physique, ou la contrainte pour surmonter une résistance. L'obésité a été définie comme un indice de masse corporelle (IMC) supérieur à $25 \mathrm{~kg} / \mathrm{m}^{2}$. L'IMC était considéré comme normal entre 18,5 et $24,9 \mathrm{~kg} / \mathrm{m}^{2}$. Les patients dont l'IMC était inférieur à $18,5 \mathrm{~kg} / \mathrm{m}^{2}$ ont été considérés comme étant maigres. Les infiltrations du canal carpien ont été réalisées avec la betamethasone, à raison d'une infiltration tous les huit jours sans dépasser un maximum de trois infiltrations.

La saisie des données a été faite avec le logiciel Epidata v3.1. L'analyse et les tests statistiques ont été réalisés avec le logiciel Epi-Info2000 v3.5.4. Pour le test du chi 2, le $\mathrm{p}$ a été considéré comme significatif lorsqu'inférieur à 0.05 .

\section{Résultats}

De 2011 à 2018, 30303 patients ont consulté dans les 3 services retenus pour l'étude. Parmi ces patients, $192(0,6 \%)$ ont consulté pour un syndrome du canal carpien. Il s'agissait de 166 femmes et 26 hommes (sexratio $\mathrm{F} / \mathrm{H} 4,8)$. L'âge moyen était de 54, $2 \pm 13$ ans (extrêmes 26 et 83 ans) pour l'ensemble des patients, de 54,6 $\pm 13,4$ ans chez les hommes, et de 54,1 $\pm 13,0$ ans chez les femmes $(\mathrm{p}=0,9)$. Les commerçantes étaient la catégorie socio-professionnelle la plus représentée (tableau 1). 
Tableau I: Répartition des patients selon leurs professions

\begin{tabular}{cccc}
\hline & Nombre $(\%)$ & $\mathrm{p}$ \\
\hline Commerçant(e)s & 73 & $(38,0)$ & 0,0001 \\
Métiers manuels & 40 & $(20,8)$ & 0,4 \\
Femme au foyer & 24 & $(12,5)$ & NA*** \\
Médecins et paramédicaux & 13 & $(6,8)$ & 0,5 \\
Enseignants & 10 & $(5,2)$ & 0,6 \\
Travailleur à l'ordinateur * & 9 & $(4,7)$ & 0,005 \\
Travailleur de force & 9 & $(4,7)$ & 0,1 \\
Autres** & 14 & $(7,3)$ & 0,00000 \\
\hline
\end{tabular}

*Travailleur prolongé à l'ordinateur: secrétaire, archiviste, attaché d'administration, comptable **Autres: ingénieur agronome, administrateur civil, agent de promotion sociale, policier, collectrice, retraité, hôtesse, interprète, religieuse $* * *$ NA: non applicable

L'indice de masse corporelle moyen était de $28,8 \pm 6,1 \mathrm{~kg} / \mathrm{m}^{2}$ (extrêmes 18 et 55,4) (tableau 2). Cinq $(2,6 \%)$ patients souffraient d'insuffisance rénale, quatre $(2,1 \%)$ étaient diabétiques, quatre $(2,1 \%)$, étaient éthyliques, trois $(1,6 \%)$ étaient porteurs du VIH, deux du virus de l'hépatite B $(1 \%)$, et un patient avait une goutte $(0,5 \%)$. Une patiente était enceinte $(0,5 \%)$. Soixante-quinze femmes $(45,1 \%$ des 166 femmes atteintes) étaient ménopausées. Aucun patient ne souffrait de polyarthrite rhumatoïde.

Tableau II: Répartition des patients selon l'indice de masse corporelle et le sexe

\begin{tabular}{ccccc}
\hline & \multicolumn{2}{c}{ Sexe } & Total $(\%)$ & $\mathrm{p}$ \\
& $\mathrm{N}=26(13,5 \%)$ & $\mathrm{N}=166(86,5 \%)$ & & \\
\hline Maigreur & 1 & 2 & $3(1,6)$ & $\mathrm{NA}^{*}$ \\
Poids normal & 13 & 36 & $49(25,5)$ & 0,002 \\
Obésité & 12 & 128 & $140(38)$ & 0,001 \\
\hline Total & 26 & 166 & $192(100)$ & \\
\hline \multicolumn{5}{c}{ *Non applicable }
\end{tabular}

Les manifestations cliniques ont été dominées par les paresthésies à type de fourmillements $(77,1 \%$ des patients), la douleur $(57,8 \%)$ et la présence d'un signe de Tinel $(56,8 \%)$ (tableau 3$)$. La presence d'un signe de Tinel était corrélé à l'obésité (tableau 4). L'atteinte était bilatérale chez 120 patients $(62,5 \%)$ et unilatérale chez 72 d'entre eux $(37,5 \%)$. Huit patients $(4,1 \%)$ ont présenté des maladresses à type de chutes d'objets, troubles de la préhension, et difficultés à écrire. Cinq patients ont présenté une hypotrophie de l'éminence Thénar, quatre patients avaient une hypoesthésie des doigts, et trois patients avaient un deficit moteur (force musculaire cotée à $3 / 5$ chez deux patients, et $4 / 5$ chez un patient). 
Tableau III : manifestations cliniques du syndrome du canal carpien

$$
\text { Effectif (pourcentage) }
$$

Douleur $(\mathrm{n}=111)$

Horaire mécanique

Horaire inflammatoire

$107(96,4)$

Horaire mixte

$3(2,7)$

$1(0,9)$

Paresthésies ( $\mathrm{n}=192)$

Fourmillements

$148(77,1)$

Engourdissements

$52(27,1)$

Décharge électrique

$2(1,0)$

Picotements

$2(1,0)$

Siège $(n=192)$

Main

Avant-bras

Poignet

$12(6,2)$

Bras

Signe de Tinel présent $(n=192)$

Signes de Tinel et de Phalen

$(n=192)$

$39(20,3)$

Tableau IV: signes de Tinel et de Phalen en fonction de l'indice de masse corporelle

\begin{tabular}{cccc}
\hline & Obèse & Non obèse & $\mathrm{p}$ \\
\hline Tinel présent & 34 & 8 & 0,001 \\
Tinel et Phalen & 75 & 31 & 0,4 \\
\hline
\end{tabular}

Cent quarante-sept patients ont bénéficié d'infiltratons de bétaméthasone (tableau 5). Un patient a reçu deux infiltrations supplémentaires après une rechute et s'est vu proposer une intervention chirurgicale. La douleur et/ou les parestésies résiduelles, après traitement, évaluées par l'échelle numérique, avait comme valeur médiane de $4 \pm 1,9$ sur dix (extrêmes 1,5 et 10). La durée moyenne d'évolution des symptômes avant la consultation était de 16,7 \pm 30 mois (extrêmes 1 et 240).

Tableau V: modalités thérapeutiques des 192 patients

\begin{tabular}{lc}
\hline & Effectif $(\%)$ \\
\hline Infiltration de corticoïde & $147(76,6)$ \\
1 infiltration & $119(61,9)$ \\
2 infiltrations & $15(7,8)$ \\
3 infiltrations & $11(5,8)$ \\
5 infiltrations & $1(0,5)$ \\
Autres traitement médicamenteux* & $104(54,2)$ \\
Kinésithérapie & $6(3,1 \%)$ \\
Indication chirurgicale & $1(0,5)$ \\
\hline
\end{tabular}

*antalgiques, AINS

\section{Discussion}

Le syndrome du canal carpien est une affection rare en consultation rhumatologique à Lomé ( $0,6 \%$ des consultations). Il touche l'adulte d'âge mûr 
(54ans) avec une prédominance féminine. Les manifestations cliniques du syndrome du canal carpien ont été dominées par les paresthésies à type de fourmillements $(77,1 \%$ des patients), la douleur $(57,8 \%$ des patients) et la présence d'un signe de Tinel (56,8\% des patients). L'atteinte était bilatérale chez 109 patients $(60,2 \%)$ et unilatérale chez 72 d'entre eux $(39,8 \%)$. Le sexe féminin, l'obésité, et le travail prolongé à l'ordinateur sont des facteurs de risque. La prise en charge repose essentiellement sur les infiltrations de corticoïdes, les antalgiques et les AINS.

L'étude a été multicentrique, réalisée dans les trois structures publiquesoù exercent des rhumatologues. Les patients pris en charge par les neurologues, les neurochirurgiens et les structures privées ont été exclus, ce qui peut générer un biais de sélection. Les informations ont été recueillies sur dossiers, d'ou le manque de précision de certaines réponses. Néanmoins ces résultats méritent d'être discutés.

Le sexe féminin est un facteur de risque de survenue d'un syndrome du canal carpien dans cette série, comme dans la littérature (Mazières, 2018; Chammas, 2014). Les changements hormonaux liés à la grossesse, l'allaitement, la ménopause, au traitement hormonal substitutif, et aux contraceptifs hormonaux en seraient la cause. La profession de commerçant est très souvent occupée par des femmes peu instruites à Lomé.

L'excès pondéral et les facteurs professionnels (mouvements répétés de flexion/extension du poignet, ou de supination de l'avant-bras, microtraumatismes liés à l'utilisation d'engins vibrants) sont d'autres facteurs de risque classiques du syndrome du canal carpien (Mazières, 2018; Aboonq, 2015; Chammas, 2014; Lee et al, 2019). Au Togo, l'obésité est socialement valorisée car considérée comme un signe d'aisance sociale chez les hommes, et un signe de beauté chez les femmes. Elle était présente chez tous les patients, mais plus marquée chez les femmes. En revanche, le rôle du travail prolongé à l'ordinateur comme un facteur de risque est controversé (Mediouni, 2015; Shiri, 2015; Thomsen, 20087).

Même si le syndrome du canal carpien est habituellement idiopathique, la recherche d'une étiologie est systématique. Il peut s'agir d'une cause locale (anomalie anatomique congénitale, fracture, luxation ou arthrite du poignet), d'une neuropathie périphérique diffuse, ou d'une maladie générale (diabète, hémodialyse, granulomatose, hypethyroïdie) (Petiot, 2011; Tahir, 2018; Pourmemari et al, 2016, Mbarki, 2013). Dans cette série, seuls neuf cas peuvent être considérés comme secondaires (cinq cas d'insuffisance rénale, quatre cas de diabète). Il faudrait à l'avenir réaliser systématiquement les examens complémentaires systématiques permettant le diagnostic de ces formes. Il faudrait également sensibiliser les sages-femmes, les gynécologues et les pédiatres pour le diagnostic de ce syndrome et l'envoi des femmes enceintes et allaitantes au rhumatologue. 
Le diagnostic a reposé exclusivement sur des arguments cliniques. En effet l'électromyogramme n'est pas disponible dans les structures publiques. L'échographie s'est imposé ces dernières années comme une alternative en raison d'une sensibilté et d'une spécificité comparables à l'électromyogramme, avec l'avantage d'être mieux tolérée, moins chère, et de permettre un bilan étiologique local. Au plan thérapeutique, elle guide les infiltrations et permet la chirurgie mini-invasive. Cependant l'échographie ne donne pas d'informations sur la sévérité de l'atteinte nerveuse. L'electromyogramme reste donc l'examen de référence pour le diagnostic (Petrover, 2018; Kotevoglu, 2005).

Les infiltrations de corticoïdes sont le moyen thérapeutique le plus utilisé en première intention.. Elles sont efficaces dans $70 \%$ des cas, avec cependant un risque de rechute (Bland, 2007). La durée d'évolution prolongée, la présence du signe de Phalen, et l'amyotrophie de l'éminence Thénar seraient des facteurs de non réponse au traitement médical pour Burton, alors pour Day, le risque d'une intervention chirurgicale augmenterait avec l'âge (Burton, 2016 ; Day, 2010). La chirurgie est envisagée en deuxième intention en cas de persistance des symptômes après quatre à six mois de traitement conservateur (Wipperman, 2016 ; Shi, 2011, Massamba, 2017). L'atteinte était accompagnée de troubles neurologiques objectifs, chez 8 patients $(4,1 \%)$, mais seul un patient s'est vu proposer la chirurgie. Les spécialistes de la chirurgie de la main ne sont présents au Togo que depuis cinq ans. Mais le faible taux de patients opérés s'explique surtout par leur réticence à la prise en charge chirurgicale, par peur des complications.

\section{Conclusion}

Le syndrome du canal carpien est rare en consultation rhumatologique à Lomé. Il est habituellement idiopathique, de topographie bilatérale, et affecte préférentiellement la femme de la cinquantaine. Les infiltrations sont efficaces. La recherche des formes secondaires doit devenir systématique afin de prendre en charge les pathologies sous-jacentes. La collaboration avec les autres professionnels de santé (sages-femmes, gynécologues, pédiatres, chirurgiens de la main) permettra de prendre en charge un plus grand nombre de femmes.

\section{References:}

1. Aboonq, MS. (2015). Pathophysiology of carpal tunnel syndrome. Neurosciences. 20: 4-9

2. Allaya, F, Ines, K, Masmoudies D, Triki, L, Zouari, H, et Masmoudi K. (2019). Tests de Tinel el Phalen : quelle sensibilité et quelle spécificité au cours du syndrome du canal carpien ? Rev Neurol. 175:S57. 
3. Aouatef, M, Henia, H, Omrane, A, Bouzgarrou, L, Amri, C, Khalfallah, T, et Henchi, A. (2016). Libération chirurgicale du syndrome de canal carpien et devenir professionnel des patients ? L'Environnement. 77 : 399.

4. Bland, JD. (2007). Treatment of carpal tunnel syndrome. Muscle Nerve. 36:167-71.

5. Burton, CL, Chesterton, LS, Chen, Y, et van der Windt, DA. (2016). Clinical Course and Prognostic Factors in Conservatively Managed Carpal Tunnel Syndrome: A Systematic Review. Arch Phys Med Rehabil 97:836-52.

6. Chammas, M, Boretto, J, Burmann, LM, Ramos RM, Santos Neto, C dos, et Silva JB. (2014). Carpal tunnel syndrome - Part I (anatomy,physiology, etiology and diagnosis). Rev Bras Ortop. 49:429-36

7. Chammas, M, Boretto, J, Burmann, LM, Ramos RM, Santos Neto, C dos, et Silva JB. (2014). Carpal tunnel syndrome - Part II (treatment). Rev Bras Ortop. 49:437-45.

8. Day, CS, Makhni, EC, Mejia, E, Lage, DE, et Rozental TD. (2010). Carpal and Cubi-tal Tunnel Syndrome. Who Gets Surgery? Clin Orthop Relat Res. 468:1796-1803.

9. Kotevoglu, N, et Gülbahce-Saglam, S. (2005). L'échographie dans le diagnostic du syndrome du canal carpien : valeur intrinsèque et comparaison avec l'évaluation clinique. Rev Rhum. 72 :221-25.

10. Lebrun-Grandié, P. (2019). Mes recettes pratiques pour explorer un syndrome du canal carpien... Rev Neurol. 175 : 154-55.

11. Lee, IH, Kim, YI, Kang,DM, Kim,SY, Kim,IA, et Kim,EM. (2019). Distribution of age, gender, and occupation among individuals with carpal tunnel syndrome based on the National Health Insurance data and National Employment Insurance data. Ann Occup Environ Med. 7;31:e31.

12. Luchetti, R, Tognon, S, Cacciavillani M, Roncos, S, Buzzellig, N, et Lanni, G. (2017). Observational multicentric survey on carpal tunnel syndrome: demographic and clinical data from 34 Italian centers. Eur Rev Med Pharmacol Sci ; 21:460-9.

13. Massamba, MD, Boukassa L, Lamini N2, Massouama R, Monka M, Ondélé N, Bileckot R, et Ntsiba H. (2017). Neurolysis of the Median Nerve to the Carpal Canal by the Way Mini-Open: Review of 68 Files at Brazzaville University Hospital. Surg Sci. 8 : 510-8.

14. Mazières, B. (2018). Syndrome du canal carpien et autres syndromes canalaires. Dans Mazières, B, Laroche, M, Constantin, A, et Cantagrel, A. Rhumatologie pour le praticien (p. 437-42). Issy-les-Moulineaux, France : Elsevier Masson. 
15. Mbarki, H, Akrichi, A, Lazrak, A, Maaroufi, C, El Midaoui A, Tachfouti N, Jellouli W, Arrayhani, M, Belahsen, MF, et Sqalli, T ; (2013). Le syndrome du canal carpien chez les patients hémodialysés chroniques. Pan Afr Med J; 14:19.

16. Mediouni, Z, Bodin J, Dale, AM, Herquelot, E , Carton, E, Leclerc, A, Fouquet, N, Dumontier, C, Roquelaure, Y, Evanoff, B A, et Descatha, A. (2015). Carpal tunnel syndrome and computer exposure at work in two large complementary cohorts. BMJ Open. 5(9):e008156.

17. Petiot, P, Bernard, E. (2011). Pièges diagnostiques du syndrome du canal carpien. Rev Neurol. 167 : 64-71.

18. Petrover, D, et Richette, P. (2018). Prise en charge du syndrome du canal carpien: de l'échographie diagnostique à la libération percutanée sous échographie. Rev Rhum. 85 :243-50

19. Pourmemari, MH, et Shiri, R. (2016). Diabetes as a risk factor for carpal tunnel syndrome: a systematic review and meta-analysis. Diabet Med. 33.

20. Shi, Q, et MacDermid, JC. (2011). Is surgical intervention more effective than non-surgical treatment for carpal tunnel syndrome? A systematic review. J Orthop Surg Res. 6:17.

21. Shin, YH, Yoon, JO, Kim, YK, et Kim, JK. (2018). Psychological Status Is Associated With Symptom Severity in Patients With Carpal Tunnel Syndrome. J Hand Surg Am. 43:484.e1-484.e8

22. Shiri, R, et Falah-Hassani, K. (2015). Computer use and carpal tunnel syndrome: A meta-analysis. J Neurol Sci. 349:15-9.

23. Tahir, A, Sdoudi, A, Chahed, M, Elbaitil, A, Fakherdine, L, Sbihi, Y, Bennouna, D, et Fadili M. (2018). Syndrome du canal carpien secondaire à une variété anatomique rare du nerf médian. Pan Afr Med J. 31,39.

24. Thomsen, JF, Gerr, F, et Atroshi, I. (2008). Carpal tunnel syndrome and the use of computer mouse and keyboard: a systematic review. BMC Musculoskelet Disord. 2008;9:134.

25. Wipperman, J, et Goerl, K. (2016). Carpal Tunnel Syndrome: Diagnosis and Management. Am Fam Physician. 94:993-9. 